\title{
The Influence of Information on Negotiation Equilibrium
}

\author{
Shaheen S. Fatima ${ }^{1} \quad$ Michael Wooldridge $^{1} \quad$ Nicholas R. Jennings $^{2}$ \\ ${ }^{1}$ Department of Computer Science, \\ University of Liverpool, Liverpool L69 7ZF, U.K. \\ \{S.S.Fatima, M.J.Wooldridge\}@csc.liv.ac.uk \\ ${ }^{2}$ Department of Electronics and Computer Science, \\ University of Southampton, Southampton SO17 1BJ, U.K. \\ nrjecs.soton.ac.uk
}

\begin{abstract}
This paper studies the influence of the agents' information states on the negotiation equilibrium. This analysis is undertaken by examining a range of negotiation scenarios in which the amount of information that agents have about their opponent's parameters is systematically varied. For each such scenario, we show that a unique equilibrium exists and we investigate how the information states of agents influence the distribution property of the equilibrium solution. Our study shows the relative impacts of the opponent's parameters on the negotiation outcome. The results obtained are useful for decision making in situations where an agent has the option of choosing whom to negotiate with, from among a set of bargainers, on the basis of its information state. Our analysis also indicates which of its opponent's parameters an agent should learn in order to maximize its utility.
\end{abstract}

\section{Introduction}

Negotiation is a means for agents to communicate and compromise to reach mutually beneficial agreements. In such situations, the agents have a common interest in cooperating, but have conflicting interests over exactly how to cooperate. Put differently, the agents can all benefit from reaching agreement on an outcome (from a set of possible outcomes), but have conflicting interests over the set of outcomes. The main problem that confronts agents in such a situation is to decide how to cooperate - before they actually cooperate and obtain the fruits of that cooperation. On the one hand, each agent would like to reach some agreement rather than disagree and not reach any agreement. But, on the other hand, each agent would like to reach an agreement that is as favourable to it as possible.

To this end, a number of negotiation models have been developed (see section 5 for more details). These models cover a broad range of agent types and environments. However to provide a focus for our work, we concentrate on one-to-one negotiations, with a deadline, between a buyer and a seller. In this type of competitive encounter, each agent has to make decisions about generating offers and counter-offers in such a way that their own utility from the final agreement is maximized. An essential input to this decision making process is information; here defined as any factor that effects the ability 
of an individual to make choices in a given situation. For instance in bargaining between a buyer and a seller, information covers the agent's own parameters (like its reservation price or its preferences over possible outcomes), as well as those of its opponent.

Existing models for bargaining with deadlines are of two types: those with complete information and those with incomplete information. In the former setting, agents know everything about each other's characteristics, as well as their own [3]. In the latter setting, agents lack information on some specific parameters about their opponent. For instance there could be uncertainty over their discounting factor [13], reservation price [4], or deadline [14]. Given such uncertainty, these models study the impact on the strategic behavior of the agents. Our objective here is not the same. Thus we do not address the issue of uncertainty per se, but rather we analyse the impact on the negotiation outcome of knowing various pieces of information about the opponent's parameters. Thus we explore a range of negotiation scenarios by varying the degree of information an agent has about its opponent.

In particular, we consider three pieces of information to be central to the way that an agent negotiates in our context. Firstly, the deadline by when the negotiation must be completed (which may be different for each party). Secondly, the time at which agreement is reached can effect the agents in different ways [2]. An agent can gain utility with time and have the incentive to reach a late agreement (within its deadline). In such a case it is said to be a patient player. Alternatively, the agent can lose utility with time and have an incentive to reach an early agreement. It is then said to be an impatient player. Thirdly, the agents' reservation limits also influence the outcome. We therefore study the effect of all these parameters on the equilibrium solution.

Against this background, we analyse the mutual strategic behavior of agents for varying degrees of information. In each of these situations, we determine equilibrium strategies and study how the information state of agents influences the distribution property of the equilibrium outcome; that is, how the gains from trade are divided between the agents. We say that an agent has more (less) bargaining power than its opponent if this division is more (less) favourable to it than to its opponent. This analysis has not previously been undertaken and so this represents the main contribution of this paper. We believe such an analysis is particularly useful for decision making in situations where an agent has the option of choosing whom to negotiate with, from among a set of bargainers, on the basis of their information state. Additionally, our results also indicate which of its opponent's parameters an agent should learn in order to maximize its utility and make it a more effective bargainer.

The remainder of the paper is structured in the following manner. Section 2 details our negotiation model. Section 3 determines the optimal and equilibrium strategies by varying the information state of the participating agents. In section 4 we compare the influence of the various negotiation parameters on the bargaining power of agents. Section 5 discusses related work. Finally in section 6 we present the conclusions and outline the avenues of further research. 


\section{The Negotiation Model}

We use an alternating offers protocol for our study. Let $b$ denote the buyer, $s$ the seller and $\left[P_{\text {min }}^{a}, P_{\text {max }}^{a}\right]$ denote the range of values for price that is acceptable to agent $a$, where $a \in\{b, s\}$. $\hat{a}$ denotes agent $a$ 's opponent. A price that is acceptable to both $b$ and $s$, i.e., the zone of agreement $(Z)$, is the interval $\left[P_{\min }^{s}, P_{m a x}^{b}\right]$. The difference between $P_{\max }^{b}$ and $P_{\min }^{s}$ is called the price-surplus. $T^{a}$ denotes agent $a$ 's deadline. Let $p_{b \rightarrow s}^{t}$ denote the price offered by agent $b$ at time $t$. Negotiation starts when the first offer is made. When an agent, say $s$, receives an offer at time $t$, i.e., $p_{b \rightarrow s}^{t}$, it rates the offer using its utility function $U^{s}$. If $U^{s}\left(p_{b \rightarrow s}^{t}, t\right)$ is greater than the utility of the counteroffer agent $s$ is ready to send at time $t^{\prime}$, i.e., $p_{s \rightarrow b}^{t^{\prime}}$ with $t^{\prime}>t$ then agent $s$ accepts. Otherwise a counter-offer is made.

Since both agents have a deadline, we assume that they use a time dependent function [1] for generating offers. These tactics vary the price depending on the remaining negotiation time, modelled as the above defined constant $T^{a}$. In these functions, the dominant factor used to decide which value to offer next is time $t$. The initial offer is a point in the interval $\left[P_{\min }^{a}, P_{\max }^{a}\right]$. Agents define a constant $k^{a}$ that when multiplied by the size of the interval determines the price to be offered in the first proposal by $a$. The offer made by $a$ at time $t\left(0<t \leq T^{a}\right)$ is defined in terms of the negotiation decision function (NDF), $F^{a}$, as follows:

$$
p_{a \rightarrow \hat{a}}^{t}= \begin{cases}P_{\min }^{a}+F^{a}(t)\left(P_{\max }^{a}-P_{\text {min }}^{a}\right) & \text { for } b \\ P_{\text {min }}^{a}+\left(1-F^{a}(t)\right)\left(P_{\text {max }}^{a}-P_{\text {min }}^{a}\right) & \text { for } s .\end{cases}
$$

A wide range of functions can be defined by varying the way in which $F^{a}(t)$ is computed (see [1] for more details). However, functions must ensure that $0 \leq F^{a}(t) \leq$ $1, F^{a}(0)=k^{a}$ and $F^{a}\left(T^{a}\right)=1$. That is, the offer will always be between the value range, at the beginning it will give the initial constant and when the deadline is reached it will offer the reservation value. Function $F^{a}(t)$ is defined as follows:

$$
F^{a}(t)=k^{a}+\left(1-k^{a}\right)\left(\frac{\min \left(t, T^{a}\right)}{T^{a}}\right)^{\frac{1}{\psi}}
$$

An infinite number of functions can be defined for different values of $\psi$. However, two extreme sets show clearly different patterns of behaviour (see Figure 1).

1. Boulware (B) [12]. For this function, $\psi<1$ and the initial offer is maintained till time is almost exhausted, when the agent concedes up to its reservation value.

2. Conceder (C) [11]. For this function, $\psi>1$ and the agent goes to its reservation value very quickly. When $\psi=1$ price is increased linearly $(\mathrm{L})$.

The value of a counter-offer depends on the initial price (IP) at which the agent starts negotiation, the final price (FP) beyond which it does not concede, $\psi$ and $T^{a}$. A vector, $\mathrm{V}$, of these four variables, i.e., $\mathrm{V}=\left[\mathrm{IP}, \mathrm{FP}, T^{a}, \psi\right]$ forms the agent's strategy. The negotiation outcome $(O)$ is an element of $\{(p, t), C\}$, where $(p, t)$ denotes the price and time at which agreement is reached and $C$ denotes the conflict outcome. 


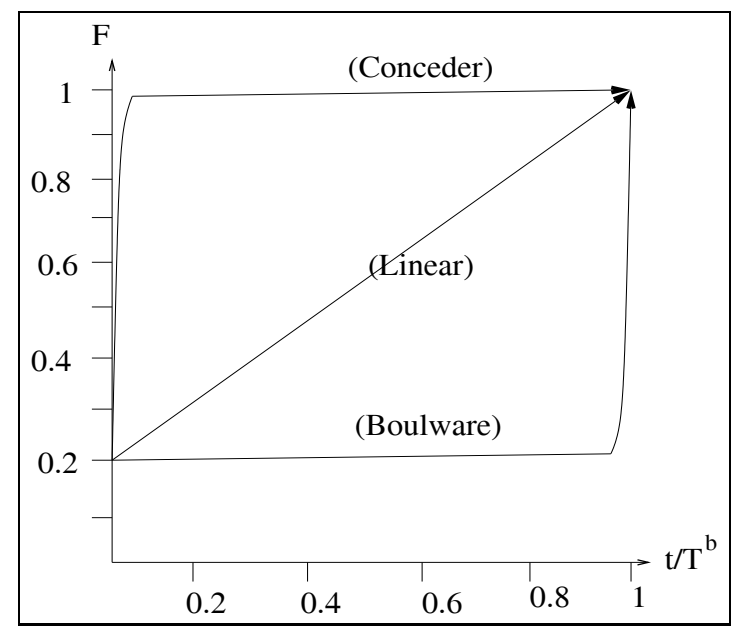

Fig. 1. Negotiation Decision Functions

When $b$ 's strategy is $\left[P_{\min }^{s}, P_{\text {max }}^{b}, T^{s}, B\right]$ and $s$ 's strategy is $\left[P_{\text {max }}^{b}, P_{\min }^{s}, T^{s}, B\right]$, the outcome $\left(O_{1}\right)$ that results is shown in Figure 2(a). As shown in the figure, agreement is reached at a price $P_{\min }^{s}+($ price-surplus $/ 2)$ and at a time close to $T^{s}$. Similarly when the NDF in both strategies is replaced with $C$, agreement is reached at the same price but near the beginning of negotiation. Figure 2(b) illustrates a negotiation conflict. The strategies for $b$ and $s$ are $\left[P_{\min }^{b}, P_{\max }^{b}, T^{b}, B\right]$ and $\left[P_{\max }^{s}, P_{\min }^{s}, T^{s}, B\right]$ respectively and $T^{s}<T^{b}$. As agents have unequal deadlines and both agents use the $B$ function, the strategies do not converge and result in a conflict. In general, agents can avoid conflict by using a strategy that offers a mutually acceptable price (i.e., within $Z$ ) by a mutually acceptable time (the earlier deadline).

Agents' utilities are defined with the following two von Neumann-Morgenstern utility functions [5] that incorporate the effects of discounting and bargaining costs:

$$
U^{a}(p, t)=U_{p}^{a}(p) U_{t}^{a}(t) \quad \text { where } \quad a \in\{b, s\}
$$

$U_{p}^{b}$ is a decreasing function of price and $U_{p}^{s}$ is an increasing function of price. For an agent, if $U_{t}^{a}$ increases with time then the agent gains utility over time and has the incentive to reach a late agreement. But if $U_{t}^{a}$ decreases with time then the agent loses with time and has the incentive to reach an early agreement. Agents are said to have similar time preferences if both gain on time or both lose on time; otherwise they have conflicting time preferences.

\section{Equilibrium Outcomes}

Each agent has a reservation limit, a deadline, and a utility function. Thus $b$ and $s$ each have three parameters, denoted $\left\{P_{\text {max }}^{b}, T^{b}, U^{b}\right\}$ and $\left\{P_{\text {min }}^{s}, T^{s}, U^{s}\right\}$ respectively. The 


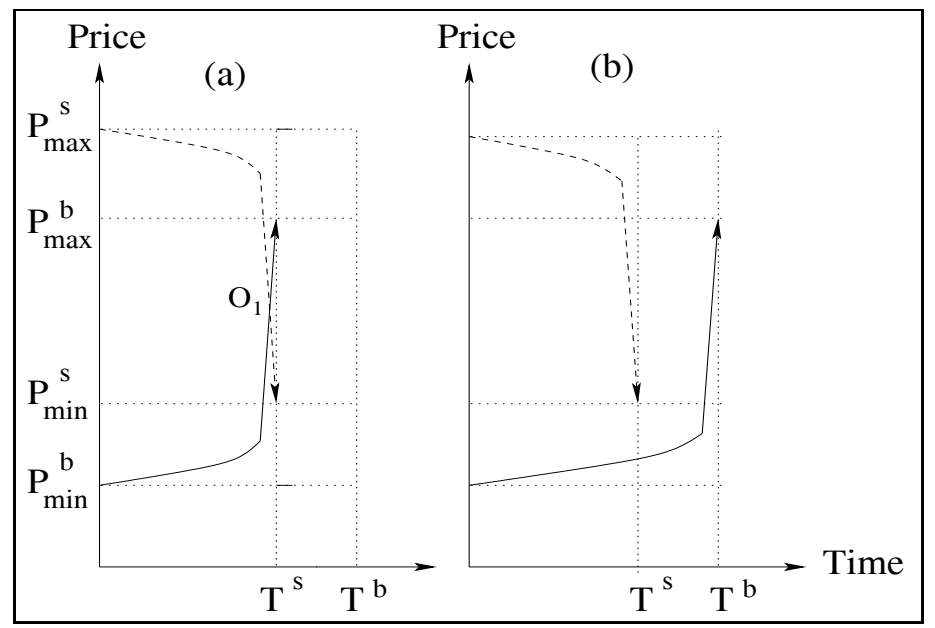

Fig. 2. Illustration of an agreement and a negotiation conflict

outcome of negotiation depends on all these six parameters. The information state of an agent is the information it has about the negotiation parameters. An agent's own parameters are known to it, but the information it has about the opponent's parameters varies. The information state $I^{a}$ of an agent $a$ is thus a set of two elements. The first element denoted $F^{a}$ is a 3-tuple containing its own parameters. This forms the fixed part of $I^{a}$. The second element, denoted $V^{a}$, is an $n$-tuple (where $n$ varies between one and three) containing information about its opponent's parameters. This forms the variable part of $I^{a}$. Thus

$$
I^{a}=\left\{F^{a}, V^{a}\right\}, F^{b}=\left\langle P_{\text {max }}^{b}, T^{b}, U^{b}\right\rangle \text { and } F^{s}=\left\langle P_{\text {min }}^{s}, T^{s}, U^{s}\right\rangle
$$

In the following subsections we vary $n$ between one and three. For each value of $n$, we determine $b$ 's optimal strategy $S^{b}$ on the basis of $I^{b}$ and $s$ 's optimal strategy $S^{s}$ on the basis of $I^{s}$ where $b$ and $s$ are von Neuman-Morgenstern expected utility maximizers. As described in the previous section, an agent's strategy is a four element vector $V=[I P, F P, T, N D F]$. This is optimal if the four elements satisfy the constraint of avoiding conflict and also result in agreement at the maximum possible utility. We then prove that this mutual strategic behavior of agents, where both use their respective optimal strategies, form sequential equilibrium points $[10,8]$.

\section{1 $V^{a}$ contains a single element}

We consider three cases where $V^{a}$ contains the opponent's deadline, reservation price and utility. For the utility of the opponent we do not take the complete function, but only its attitude towards time. This is because, in practice, it is easier for an agent to know its opponent's attitude towards time rather than the entire utility function. 


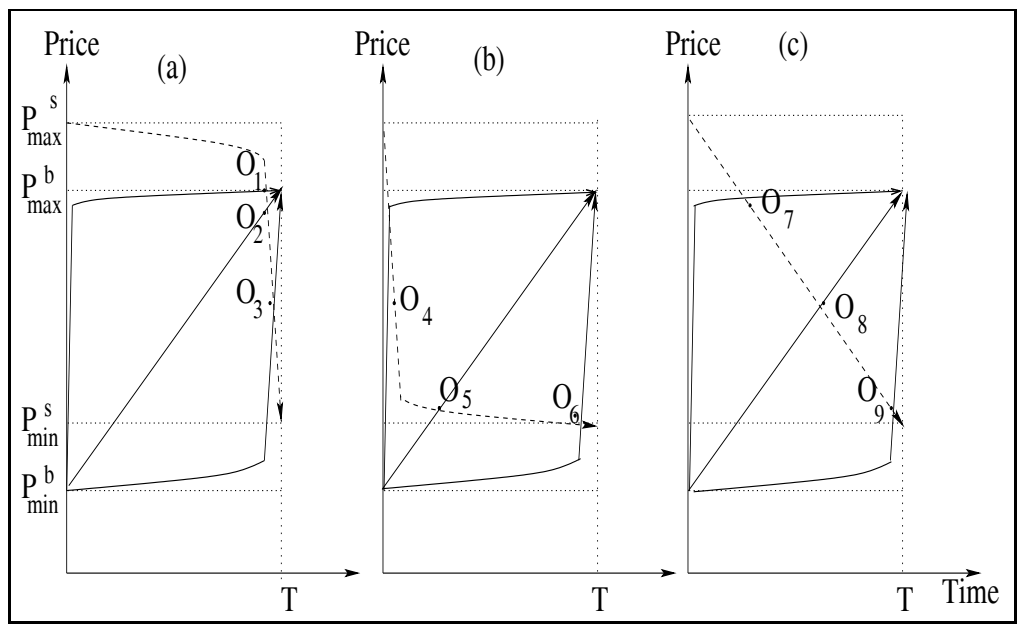

Fig. 3. Possible strategies and associated outcomes

1. Deadline. When agents know each others' deadline, the information states are $V^{b}=\left\{T^{s}\right\}$ and $V^{s}=\left\{T^{b}\right\}$.

Optimal strategies. In the absence of $P_{\text {min }}^{s}, b$ can ensure convergence by making $I P=P_{\min }^{b}$ (a very low price that lies outside $\left.Z\right), F P=P_{\text {max }}^{b}$, and offering $F P$ before the earlier deadline. Thus the third element of the strategy becomes $T^{s}$ if $\left(T^{s}<T^{b}\right)$ and $T^{b}$ if $\left(T^{b}<T^{s}\right)$. Given this, the last element, i.e., the NDF, that optimises the time of agreement needs to be determined. Figure 3 depicts the negotiation outcome for each of the three NDFs. Note that in this figure the Boulware function is an extreme Boulware and the Conceder function is similarly extreme. $T$ denotes the earlier deadline. The dashed lines indicate $s$ 's strategy and the solid lines indicate $b$ 's strategy. Note that the actual values of IP and FP in $s$ 's strategy are not known to $b$. IP is taken as some value greater than $P_{\max }^{b}$ and FP as some value less than $P_{\max }^{b}$. Out of the three NDFs we need to determine the one that always gives $b$ the best possible utility. $b$ can have two possible attitudes towards time. It can gain utility with time and have the incentive to reach a late agreement or it can lose utility with time and have the incentive to reach an early agreement. Consider the case where $b$ gains on time. If $s$ uses the Boulware NDF, then, as seen in Figure 3(a), the outcome can be $O_{1}, O_{2}$ or $O_{3}$ depending on $b$ 's strategy. Out of these three, $O_{3}$ results in agreement at the lowest price and highest time. Thus if $s$ uses the Boulware NDF, it is best for $b$ to also use the Boulware NDF. Similarly if $s$ uses the Conceder (or Linear) NDF, (see Figures 3(b) and 3(c)) the most favourable outcome to $b$ is $O_{6}$ (or $O_{9}$ ) generated by the Boulware NDF. Thus if $b$ gains on time, irrespective of $s$ 's strategy, it is best for it to always use the B NDF. $b$ 's optimal strategy is therefore $S^{b}=\left[P_{\min }^{b}, P_{\max }^{b}, T, B\right]$. Consider the other possibility where $b$ loses utility on time. Here we consider scenarios where $Z$ is small and $T$ is large. So the gain in utility in time from $O_{5}$ to $O_{4}$ (and $O_{6}$ to $O_{5}$ ) outweighs the 
loss in utility from price. In other words, agents always try to minimize the time of agreement as long as the price is within the zone of agreement. As shown in Figures 3(a) 3(b) and 3(c), irrespective of $s$ 's strategy, $b$ can minimize the time of agreement by using the Conceder NDF. $b$ 's optimal strategy therefore becomes $S^{b}=\left[P_{\min }^{b}, P_{\max }^{b}, T, C\right]$. Analogously, $S^{s}=\left[P_{\max }^{s}, P_{\min }^{s}, T, B / C\right]$ where $P_{\max }^{s}$ is some high price outside $Z$ and the last element in $S^{s}$ is $B$ if $s$ gains on time and $C$ if it loses on time.

Since an agent's optimal strategy does not depend on its opponent's strategy, neither agent has the incentive to deviate from it at any point during negotiation. We now prove that this mutual strategic behavior of agents forms a sequential equilibrium point. As agents do not have information about their opponent's strategy or utility, negotiation can be considered as a game $\mathbf{G}$ of incomplete information. A strategy profile and belief system pair is a sequential equilibrium of an extensive game if it is sequential rational and consistent [8]. A system of beliefs $\mu$ in $\mathbf{G}$ is a specification of a probability $x \in[0,1]$ for each decision node $x$ in $\mathbf{G}$ such that

$$
\sum_{x \in \mathbf{I}} \mu(x)=1
$$

for all information sets $\mathbf{I}$. In other words, $\mu$ represents the agent's beliefs about the history of negotiation. The player's strategies satisfy sequential rationality if for each information set of each player $a$, the strategy of player $a$ is a best response to the other player's strategies, given $a$ 's beliefs in that information set. The requirement for $\mu$ to be consistent with the strategy profile is as follows. Even at an information set that is not reached if all players adhere to their strategies, it is required that a player's belief be derived from some strategy profile using Bayes' rule.

Proposition 1. There exists sequential equilibrium of $\mathbf{G}$ at the point $S^{b} \times S^{s}$.

Proof. The first three levels of the extensive form for this game $(\mathbf{G})$ are shown in Figure 4. At node 1, one of the players, say $b$, starts negotiation using its optimal strategy $S^{b}$. After the first offer, play reaches node 2. At this level, it is player $s$ 's turn to make a decision. $\mathbf{I}_{1}$ becomes the information set for $s$ since it is unaware of the strategy used by $b$ and hence does not know which of the three nodes 2, 3 or 4 , play has reached. However, irrespective of exactly which node play reaches at this level (i.e., irrespective of $s$ 's belief about the history of negotiation), the dominant strategy for $s$ is $S^{s}$. Play now reaches node 5 (since both agents use B) at which $b$ makes a move. At this point $b$ does not know exactly which node the play is at, but it knows that its information set $\mathbf{I}_{2}$ is reached with probability 1 (probability of reaching other decision nodes at this level is 0 ). The dominant strategy for $b$ at this information set (and at all others) is $S^{b}$. Thus at every information set at which it is $b$ 's turn to move, its optimal strategy is $S^{b}$ and at every information set at which it is $s$ 's turn to make a move, its optimal strategy is $S^{s}$. The strategy profile $S^{b} \times S^{s}$ therefore satisfies the requirements for sequential rationality. Furthermore, at every information set the optimal strategies are also dominant strategies. This makes the strategy profile $S^{b} \times S^{s}$ a sequential equilibrium point irrespective of the agents' 


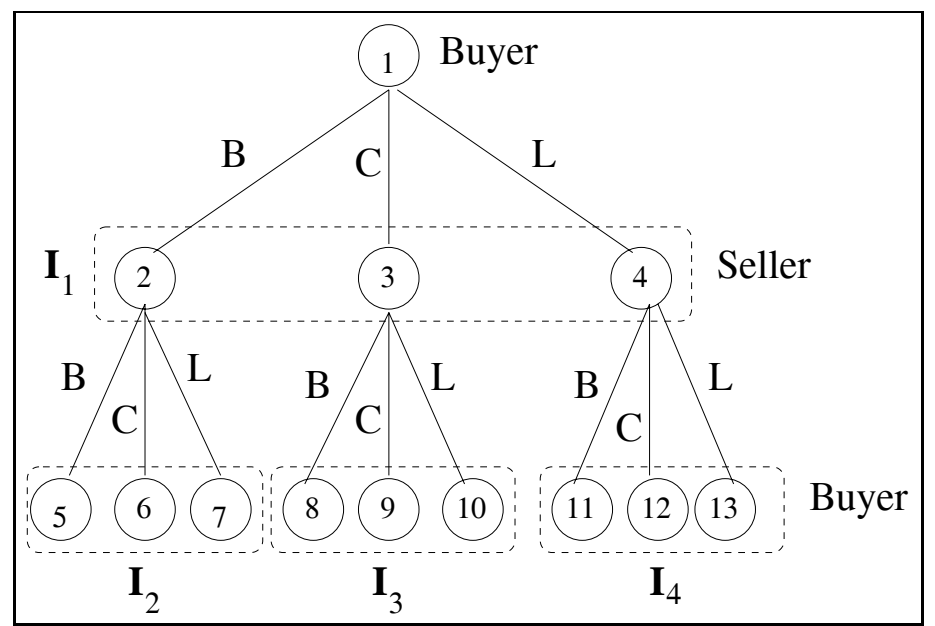

Fig. 4. Extensive form of the negotiation game

beliefs about the history of negotiation.

Corollary 1. The equilibrium at $S^{b} \times S^{s}$ is unique.

This is a direct consequence of the above proof. As the optimal strategies for both agents are dominant strategies at each of their information sets, there does not exist any other equilibrium (neither a pure nor a mixed strategy) where an agent uses a strategy other than its optimal strategy. The equilibrium solution is therefore unique.

The equilibrium outcomes for $V^{a}=\left\{T^{\hat{a}}\right\}$ in the four possible negotiation scenarios are listed in table 1.

2. Reservation price $\left(R P^{a}\right)$. Here $V^{b}=\left\{P_{\text {min }}^{s}\right\}$ and $V^{s}=\left\{P_{\text {max }}^{b}\right\}$. In the absence of $T^{s}, b$ can ensure convergence if it starts making offers at $P_{\min }^{s}$ and reaches $P_{\max }^{b}$ by $T^{b}$. Using the same analysis as for $V^{a}=\left\{T^{\hat{a}}\right\}$, we get $S^{b}=\left[P_{\min }^{s}, P_{\max }^{b}, B / C, T^{b}\right]$. The third element is B if $b$ is patient and C if it is impatient. Similarly the optimal strategy for $s$ becomes $S^{s}=\left[P_{m a x}^{b}, P_{m i n}^{s}, B / C, T^{s}\right]$. The proof of proposition 1 can also be used to show that a unique equilibrium exists at $S^{b} \times S^{s}$ in this case. The equilibrium outcomes for $V^{a}=\left\{R P^{\hat{a}}\right\}$ are also listed in table 1 .

3. Attitude towards time $\left(A^{a}\right)$. Here $V^{b}=\left\{A^{s}\right\}$ and $V^{s}=\left\{A^{b}\right\}$ and agents know whether the opponent's utility increases or decreases with time ( $\mathrm{G}$ or $\mathrm{L}$ ) but not the complete utility function. In the absence of any other information about the opponent, the strategies available to the agents are $S^{b}=\left[P_{\min }^{s}, P_{\max }^{b}, B / C, T^{b}\right]$ and $S^{s}=\left[P_{\text {max }}^{s}, P_{\text {min }}^{s}, B / C, T^{s}\right]$. However as illustrated in Figure $2(\mathrm{~b})$, these strategies do not ensure agreement and can result in conflict. 


\begin{tabular}{|c|c|c|c|c|}
\hline \multirow[t]{2}{*}{$\overline{V^{a}}$} & \multirow[t]{2}{*}{$\mathrm{b}, \mathrm{s}$} & \multirow{2}{*}{$\begin{array}{c}\text { Equilibrium } \\
\text { outcome }\end{array}$} & \multicolumn{2}{|c|}{ Bargaining Power } \\
\hline & & & Price & Time \\
\hline$\overline{\overline{T^{\hat{a}}}}$ & G,G & $\overline{(\overline{(T, P)}}$ & $\left(\boldsymbol{P}^{b}=\boldsymbol{P}^{s}\right)$ & $\overline{\overline{\left(\boldsymbol{P}^{b}=\boldsymbol{P}^{s}\right)}}$ \\
\hline$\overline{T^{a}}$ & $\mathrm{~L}, \mathrm{~L}$ & $\left(T_{0}, P\right)$ & $\boldsymbol{P}^{b}=\boldsymbol{P}^{s}$ & $\left(\boldsymbol{P}^{b}=\boldsymbol{P}^{s}\right)$ \\
\hline$\overline{T^{\hat{a}}}$ & $\mathrm{G}, \mathrm{L}$ & $\overline{\left(T, P_{\text {min }}^{s}\right)}$ & $\overline{\left(\boldsymbol{P}^{b}>\boldsymbol{P}^{s}\right)}$ & $\left(\boldsymbol{P}^{b}>\boldsymbol{P}^{s}\right)$ \\
\hline$\overline{T^{a}}$ & $\mathrm{~L}, \mathrm{G}$ & $\left(T, P_{\max }^{b}\right)$ & $\left(\boldsymbol{P}^{s}>\boldsymbol{P}^{b}\right)$ & $\left(\boldsymbol{P}^{s}>\boldsymbol{P}^{b}\right)$ \\
\hline$\overline{\overline{R P^{\hat{a}}}}$ & $\mathrm{G}, \mathrm{G}$ & $\begin{array}{l}\left(T, P_{\min }^{s}\right) \dagger \\
\left(T, P_{\max }^{b}\right) \ddagger\end{array}$ & $\begin{array}{l}\left(\boldsymbol{P}^{b}>\boldsymbol{P}^{s}\right) \dagger \\
\left(\boldsymbol{P}^{s}>\boldsymbol{P}^{b}\right)\end{array}$ & $\overline{\overline{\left(\boldsymbol{P}^{b}=\boldsymbol{P}^{s}\right)}}$ \\
\hline$R P^{a}$ & $\mathrm{~L}, \mathrm{~L}$ & $\overline{\left(T_{0}, P\right)}$ & $\left(\boldsymbol{P}^{b}=\boldsymbol{P}^{s}\right)$ & $\left(\boldsymbol{P}^{b}=\boldsymbol{P}^{s}\right)$ \\
\hline$R P^{a}$ & $\mathrm{G}, \mathrm{L}$ & $\left(T_{0}, P_{\text {min }}^{s}\right)$ & $\left(\boldsymbol{P}^{b}>\boldsymbol{P}^{s}\right)$ & $\left(\boldsymbol{P}^{s}>\boldsymbol{P}^{b}\right)$ \\
\hline$R P^{a}$ & $\mathrm{~L}, \mathrm{G}$ & $\left(T_{0}, P_{\max }^{b}\right)$ & $\left(\boldsymbol{P}^{s}>\boldsymbol{P}^{b}\right)$ & $\left(\boldsymbol{P}^{b}>\boldsymbol{P}^{s}\right)$ \\
\hline $\begin{array}{l}T^{\hat{a}}, \\
R P^{\hat{a}}\end{array}$ & $\mathrm{G}, \mathrm{G}$ & $\begin{array}{l}\left(T, P_{\min }^{s}\right) \dagger \\
\left(T, P_{\max }^{b}\right) \dagger\end{array}$ & $\begin{array}{l}\left(\boldsymbol{P}^{b}>\boldsymbol{P}^{s}\right) \\
\left(\boldsymbol{P}^{s}>\boldsymbol{P}^{b}\right)\end{array}$ & $\overline{\left(\boldsymbol{P}^{b}=\boldsymbol{P}^{s}\right)}$ \\
\hline $\begin{array}{c}T^{\hat{a}} \\
R P^{\hat{a}}\end{array}$ & $\mathrm{~L}, \mathrm{~L}$ & $\begin{array}{l}\left(T_{0}, P_{\text {min }}^{s}\right) \dagger \\
\left(T_{0}, P_{\text {max }}^{b}\right) \ddagger\end{array}$ & $\left(\begin{array}{l}\left.\boldsymbol{P}^{b}>\boldsymbol{P}^{s}\right) \\
\left(\boldsymbol{P}^{s}>\boldsymbol{P}^{b}\right)\end{array}\right.$ & $\left(\boldsymbol{P}^{b}=\right.$ \\
\hline $\begin{array}{l}T^{\hat{a}} \\
R P^{\hat{a}}\end{array}$ & $\mathrm{G}, \mathrm{L}$ & $\begin{array}{l}\left(T, P_{\min }^{s}\right) \dagger \\
\left(T, P_{\max }^{b}\right) \ddagger\end{array}$ & $\left(\begin{array}{l}\left.\boldsymbol{P}^{b}>\boldsymbol{P}^{s}\right) \\
\left(\boldsymbol{P}^{s}>\boldsymbol{P}^{b}\right)\end{array}\right.$ & $\left(\boldsymbol{P}^{b}>\boldsymbol{P}^{s}\right)$ \\
\hline $\begin{array}{l}T^{\hat{a}} \\
R P^{\hat{a}}\end{array}$ & $\mathrm{~L}, \mathrm{G}$ & $\begin{array}{l}\left(T, P_{\min }^{s}\right) \dagger \\
\left(T, P_{\max }^{b}\right) \dagger\end{array}$ & $\left(\begin{array}{l}\left.\boldsymbol{P}^{b}>\boldsymbol{P}^{s}\right) \\
\left(\boldsymbol{P}^{s}>\boldsymbol{P}^{b}\right)\end{array}\right.$ & $(\boldsymbol{P}$ \\
\hline
\end{tabular}

Table 1. Equilibrium outcomes and bargaining power for different $V^{a} \mathrm{~s}$. G indicates that the agent gains on time and $\mathrm{L}$ that it loses on time. $T_{0}$ denotes the beginning of negotiation, $T$ the earlier deadline, $P$ denotes $\left(\frac{P_{\min }^{s}+P_{\max }^{b}}{2}\right), \dagger$ indicates the outcome if $T^{s}<T^{b}$ and $\ddagger$ if $T^{b}<T^{s}$.

\section{2 $V^{a}$ contains two elements}

We now consider the effect of different parameter pairs on the negotiation equilibrium.

1. Deadline, Reservation price. Here $V^{b}=\left\{T^{s}, P_{\min }^{s}\right\}$ and $V^{s}=\left\{T^{b}, P_{\max }^{b}\right\}$. With this information available to both agents, the optimal strategies can be determined using backward induction as follows. Consider the case where $\left(T^{s}<T^{b}\right)$ as shown in Figure 5. The thick line denotes $b$ 's strategy and the dashed lines denote $s$ 's strategy. No matter which strategy $s$ uses, it is bound to reach $P_{\min }^{s}$ by $T^{s}$ since it would have to quit if agreement is not reached by $T^{s} . b$ can use this information to maximize its utility by never offering a price more than $P_{\min }^{s}$ prior to $T^{s}$. If $b$ gains on time, its optimal strategy, $S^{b}$, is $\left[P_{\min }^{b}, P_{\min }^{s}, B, T^{s}\right]$ since B has the property of not reaching $P_{\min }^{s}$ before $T^{s}$. On the other hand if $b$ loses on time, it tries to reach an early agreement and $S^{b}$ becomes $\left[P_{m i n}^{b}, P_{m i n}^{s}, C, T^{s}\right]$. The optimal strategy for $s$, $S^{s}$, will be $\left[P_{\max }^{s}, P_{\min }^{s}, B, T^{s}\right]$ if it gains utility with time or $\left[P_{\max }^{s}, P_{\min }^{s}, C, T^{s}\right]$ if it loses utility with time. In the other case where $\left(T^{b}<T^{s}\right)$, $s$ will maximize its utility by never offering a price lower than $P_{\max }^{b}$ before $T^{b}$. Thus $S^{s}$ is $\left[P_{\max }^{s}, P_{\max }^{b}, B, T^{b}\right]$ if it gains on time, and $\left[P_{\max }^{s}, P_{\max }^{b}, C, T^{b}\right]$ if it loses on time. $S^{b}$ is $\left[P_{\text {min }}^{b}, P_{\text {max }}^{b}, B, T^{b}\right]$ if it gains on time and $\left[P_{\text {min }}^{b}, P_{\text {max }}^{b}, C, T^{b}\right]$ if it loses on time. Here again the proof of proposition 1 can be used to show the existence of a 


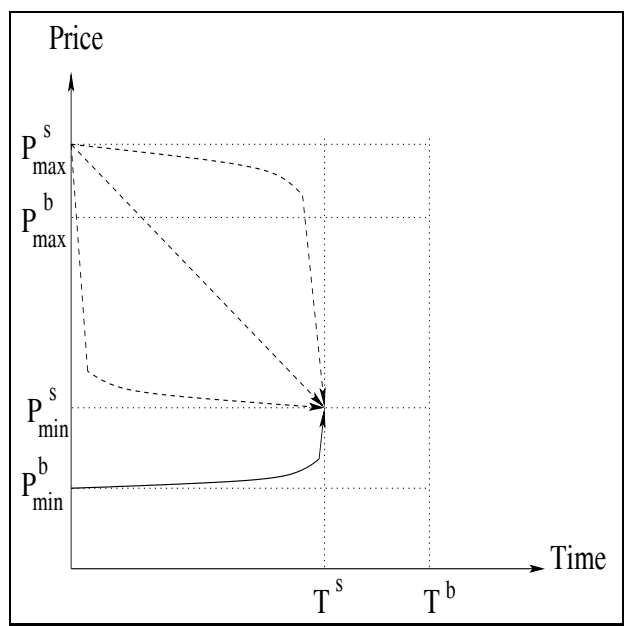

Fig. 5. Possible seller strategies

unique equilibrium at $S^{b} \times S^{s}$. The equilibrium outcomes for $V^{a}=\left\{T^{\hat{a}}, R P^{\hat{a}}\right\}$ are listed in table 1.

2. Reservation price, Attitude towards time. Here $V^{b}=\left\{P_{\min }^{s}, A^{s}\right\}$ and $V^{s}=\left\{P_{\max }^{b}, A^{b}\right\}$. An agent's optimal strategy when $V^{a}=\left\{R P^{\hat{a}}\right\}$ is independent of its opponent's strategy. Adding the opponent's attitude towards time to $V^{a}$ therefore gives the same equilibrium strategies and outcomes as for $V^{a}=\left\{R P^{\hat{a}}\right\}$.

3. Deadline, Attitude towards time. As in the previous case, this gives the same equilibrium outcomes as for $V^{a}=\left\{T^{\hat{a}}\right\}$.

\section{3 $V^{a}$ contains three elements}

Here $V^{b}=\left\{T^{s}, P_{\text {min }}^{s}, A^{s}\right\}$ and $V^{s}=\left\{T^{b}, P_{\text {max }}^{b}, A^{b}\right\}$. The optimal strategies of agents for $V^{a}=\left\{T^{\hat{a}}, R P^{\hat{a}}\right\}$ do not depend on the opponent's attitude towards time (see section for $n=2$ ). This gives the same equilibrium outcomes as for $V^{a}=\left\{T^{\hat{a}}, R P^{\hat{a}}\right\}$. But $A^{\hat{a}}$ can be used to infer the opponent's NDF. For $(n=1)$ and $(n=2)$ we showed that a patient player uses the Boulware NDF and an impatient player uses the Conceder NDF. This makes $\mathbf{G}$ a game of perfect information and both agents can pre-compute the negotiation outcome with the available information. The outcome is the same as the one obtained without $A^{a}$ (see table 1) but its inclusion eliminates the need for agents to go through the process of negotiation to arrive at it.

\section{Agents' Bargaining Power}

The distribution property of a negotiation outcome relates to the issue of how the gains from trade are divided between the players. The price $P_{e}$ and time $T_{e}$ of the equilibrium agreement reflects the relationship between the agents' bargaining powers. We say that 
an agent has more (less) bargaining power than its opponent if $\left(P_{e}, T_{e}\right)$ is more (less) in its favour than its opponent. Assume that the price-surplus is split between $b$ and $s$ in the ratio $x: y$. The agent $b$ is said to have more (less) power over price if $x>y(x<y)$. In other words, an agent's bargaining power is determined on the basis of its share of the price-surplus. Regarding the time of agreement, if $b$ prefers an early agreement but $s$ prefers a late agreement and the actual time of agreement is the earlier deadline, then $s$ is said to have more bargaining power over time. Note that we do not use the agents' utility functions to determine their bargaining power since these functions can be subjective. We now study the influence of the agents' information about their opponent's parameters on the bargaining power. These results are summarised in table 1.

1. Influence of opponent's deadline. When agents know each others' deadline, the patient agent has equal or more power than its opponent over both price and time. This can be explained as follows. Consider first the case where agents have similar time preferences. The price-surplus is divided equally between the agents (see previous section) giving them equal power over price. When both gain on time, agreement is reached at the earlier deadline and when both lose on time, agreement is reached towards the beginning of negotiation. In other words, the time of agreement is as favourable as possible to both agents giving them equal power over time. When agents have conflicting time preferences, the entire price-surplus goes to the patient agent and agreement is reached at the earlier deadline. This happens because although the impatient player attempts to reach an early agreement by using the Conceder NDF, its opponent's strategy delays agreement till the earlier deadline. Thus both $P_{e}$ and $T_{e}$ are in favour of the patient agent giving it more power than its opponent.

2. Influence of opponent's reservation-price. When agents know each others' reservation price, the patient agent has either more or less power than its opponent and the impatient agent has equal or less power than its opponent over price. With respect to time, the patient agent has equal or less power than its opponent and the impatient agent has equal or more power than its opponent. This can be explained as follows. Consider similar time preferences first. When both gain on time, the price-surplus goes to the agent with the longer deadline giving it more power than its opponent. When both lose on time, the price-surplus is divided equally between the agents giving them equal power over $P_{e}$. Agreement is reached at the earlier deadline when both gain on time and at the beginning of negotiation when both lose on time. Thus $T_{e}$ is as favourable as possible to both agents giving them equal power. When agents have conflicting time preferences, the price-surplus goes to the patient agent and agreement is reached towards the beginning of negotiation. This happens because the initial offers are $P_{\min }^{s}$ for $b$ and $P_{\max }^{b}$ for $s$. The time of agreement is in favour of the impatient agent. Thus the patient agent has more power over $P_{e}$ but the impatient agent has more power over $T_{e}$.

3. Influence of deadline and reservation-price. When agents know each others' deadline and reservation price, the agent with the longer deadline always has more power than its opponent over price. An agent's power over time depends on its attitude towards time. The patient agent has equal or more power than its opponent over time. This parameter combination always gives the entire price-surplus 
to the agent with the longer deadline giving it more power over price. Agreement is reached at the earlier deadline when at least one agent gains on time and at the beginning of negotiation when both lose on time. Thus agents have equal power in the case of like time preferences, but the patient agent gets more power in the case of conflicting time preferences.

4. Influence of opponent's attitude towards time. Adding this information when there is no existing information, or when there is information on any other single parameter (or a parameter pair) doesn't alter the equilibrium strategies or the outcome. However adding this information in addition to both deadline and reservation price eliminates the need for negotiation between agents as the solution can be pre-computed.

We now illustrate how the results from table 1 can be used when $b$ has the option of choosing between two sellers, $s_{1}$ and $s_{2}$, on the basis of its information state. Consider the case where agents know each others' deadlines and reservation prices. Assume that $b$ gains on time and $\left(T^{s_{1}}<T^{s_{2}}<T^{b}\right)$. The equilibrium outcome is $\left(T^{s_{1}}, P_{\text {min }}^{s_{1}}\right)$ if $b$ negotiates with $s_{1}$ and $\left(T^{s_{2}}, P_{\text {min }}^{s_{2}}\right)$ if $b$ negotiates with $s_{2}$. If $\left(U^{b}\left(T^{s_{1}}, P_{\text {min }}^{s_{1}}\right)>\right.$ $\left.U^{b}\left(T^{s_{1}}, P_{\min }^{s_{1}}\right)\right)$, then $b$ has more bargaining power over $s_{1}$ than it has over $s_{2}$, i.e., $\left(\boldsymbol{P}_{s_{1}}^{b}>\boldsymbol{P}_{s_{2}}^{b}\right)$ and it therefore chooses $s_{1}$. If $\left(U^{b}\left(T^{s_{1}}, P_{\text {min }}^{s_{1}}\right)<U^{b}\left(T^{s_{1}}, P_{\text {min }}^{s_{1}}\right)\right)$, then $\left(\boldsymbol{P}_{s_{1}}^{b}>\boldsymbol{P}_{s_{2}}^{b}\right)$ and $b$ chooses $s_{2}$.

Consider another case where $b$ and $s_{1}$ know each other's deadlines and $b$ and $s_{2}$ know each other's deadlines and reservation prices. $b$ 's equilibrium outcome with $s_{1}$ is $\left(T^{s_{1}}, \frac{P_{\min }^{s_{1}}+P_{\max }^{b}}{2}\right)$ if $s_{1}$ gains on time and $\left(T^{s_{1}}, P_{m i n}^{s_{1}}\right)$ if $s_{1}$ loses on time. On the other hand, the equilibrium outcome between $b$ and $s_{2}$ is always $\left(T^{s_{2}}, P_{\min }^{s_{2}}\right)$ and $b$ gets the entire price-surplus. From $s_{1}, b$ gets only half the price-surplus if $s_{1}$ gains on time. Also since $b$ is a patient player, it prefers $T^{s_{2}}$ to $T^{s_{1}}$. Thus $\boldsymbol{P}_{s_{2}}^{b}>\boldsymbol{P}_{s_{1}}^{b}$.

In terms of finding which parameter an agent should learn about its opponent, consider the example of an impatient agent. In this case, both $b$ and $s$ can be better off if they learn $R P^{\hat{a}}$, rather than $T^{\hat{a}}$ or $A^{\hat{a}}$ (see table 1 ). Thus out of the three, the most crucial piece of information for an impatient agent to learn is the opponent's reservation price $R P^{\hat{a}}$.

\section{Related Work}

A number of strategic models have been developed to explain the deadline effect on the bargaining outcome. These can be separated into models with complete information and those with incomplete information. The recent ones among the complete information models include [3] and [9]. Fershtman and Seidmann model deadline effects in a multiperiod sequential bargaining model in which the player who will propose in each period is chosen by a lottery. Equilibrium behavior in this model depends on the discount factor; if it is low, agreement is reached in the first period, but if it is high enough, then the game will end in the last period with the proposer receiving all the surplus. Ma and Manove's model is also one of complete information but with imperfect player control over the timing of the offers. Agreements in this model tend to be made near the deadline and the division of surplus is close to an even split. In contrast to this, 
in our model, when there is complete information, the time of agreement is $T_{0}$ when both agents are impatient and $T$ otherwise. Note that the time of agreement does not depend on the actual discounting factor. The entire price-surplus goes to the agent with the longer deadline.

Turning now to the incomplete information models: $[7,6]$ is a study of the strategic behaviour of agents when there is incomplete information about each other's utility functions. [13] models uncertainty over the player's discounting factors. [4] analyses bargaining in which reservation prices are uncertain, [14] considers uncertainty over agent deadlines and [2] addresses uncertainty over both deadlines and reservation prices. These existing models study the strategic behavior of agents by considering a particular scenario in which there is uncertainty over a chosen negotiation parameter. Our aim, on the other hand, is to determine the relative influences of such parameters on the negotiation outcome for a model based on negotiation decision functions. We therefore determine equilibrium outcomes for a range of scenarios by varying the information state of agents. On the basis of equilibrium agreements, we determine their bargaining power.

\section{Conclusions}

In this paper we analysed the process of bilateral negotiation by varying the information state of agents. We determined equilibrium points for a number of scenarios and analysed the relative influences of negotiation parameters on the equilibrium outcome. Our study reveals that for any negotiation scenario, there are two possible values for the time of the equilibrium agreement: the beginning of negotiation or the earlier deadline. The price of the equilibrium agreement also has only two possible values. Either the entire price-surplus goes to a single agent or both agents get an equal share of it. On the basis of equilibrium agreements we determined the relationship between agents' bargaining powers.

In our present study, the information of both agents was symmetric, i.e., both agents had information on the same parameter(s) about each other. In future we will extend our analysis to determine negotiation equilibria where agents have unequal amounts and types of information. For instance, the buyer has information about the seller's deadline or its reservation price but the seller has no information about the buyer.

\section{Acknowledgements}

This research was supported by the EPSRC under grant GR/M07052.

\section{References}

1. P. Faratin, C. Sierra, and N. R. Jennings. Negotiation decision functions for autonomous agents. International Journal of Robotics and Autonomous Systems, 24(3-4):159-182, 1998.

2. S. S. Fatima, M. Wooldridge, and N. R. Jennings. Optimal negotiation strategies for agents with incomplete information. In ATAL-2001, pages 53-68, Seattle, USA, 2001. 
3. C. Fershtman and D. J. Seidmann. Deadline effects and inefficient delay in bargaining with endogenous commitment. Journal of Economic Theory, 60(2):306-321, 1993.

4. D. Fudenberg, D. Levine, and J. Tirole. Infinite horizon models of bargaining with one sided incomplete information. In A. Roth, editor, Game Theoretic Models of Bargaining. University of Cambridge Press, Cambridge, 1985.

5. R. Keeney and H. Raiffa. Decisions with Multiple Objectives: Preferences and Value Tradeoffs. New York: John Wiley, 1976.

6. S. Kraus. Startegic negotiation in multiagent environments. The MIT Press, Cambridge, Massachusetts, 2001.

7. S. Kraus, J. Wilkenfeld, and G. Zlotkin. Multiagent negotiation under time constraints. Artificial Intelligence Journal, 75(2):297-345, 1995.

8. D. M. Kreps and R. Wilson. Sequential equilibrium. Econometrica, 50:863-894, 1982.

9. C. A. Ma and M. Manove. Bargaining with deadlines and imperfect player control. Econometrica, 61:1313-1339, 1993.

10. M. J. Osborne and A. Rubinstein. A Course in Game Theory. The MIT Press, 1998.

11. D. G. Pruitt. Negotiation Behavior. Academic Press, 1981.

12. H. Raiffa. The Art and Science of Negotiation. Harvard University Press, Cambridge, USA, 1982.

13. A. Rubinstein. A bargaining model with incomplete information about time preferences. Econometrica, 53:1151-1172, January 1985.

14. T. Sandholm and N. Vulkan. Bargianing with deadlines. In AAAI-99, pages 44-51, Orlando, FL, 1999. 